Published in final edited form as:

J Dent Res. 2005 December ; 84(12): 1154-1159.

\title{
Does Periodontal Treatment Improve Glycemic Control in Diabetic Patients? A Meta-analysis of Intervention Studies
}

\author{
S.-J. Janket ${ }^{1,{ }^{*}, 2}$, A. Wightman ${ }^{3}$, A.E. Baird ${ }^{4}$, T.E. Van Dyke ${ }^{5}$, and J.A. Jones ${ }^{1,6}$ \\ 1 Department of General Dentistry, Boston University, Goldman School of Dental Medicine, 100 \\ East Newton Street, Boston, MA 02118, USA; \\ 2 Harvard School of Public Health, Department of Nutrition, Boston, MA, USA; \\ 3 US Air Force 59th Dental Squadron, San Antonio, TX, USA; \\ 4 Stroke Neuroscience Unit, NINDS, National Institutes of Health, Bethesda, MD, USA; \\ 5 Clinical Research Center/Periodontology and Oral Biology, Boston University Goldman School of \\ Dental Medicine, Boston, MA, USA; and
}

6 VA Center for Health Quality, Outcomes and Economic Research, Bedford, MA, USA

\begin{abstract}
Previous analyses regarding effects of periodontal treatment on glycemic control included studies where causal association might not be assumed, or the results were reported non-quantitatively. We initiated this meta-analysis of 10 intervention studies to quantify the effects of periodontal treatment on $\mathrm{HbA} 1 \mathrm{c}$ level among diabetic patients, to explore possible causes for the discrepant reports, and to make recommendations for future studies. Data sources were MEDLINE (January, 1980, to January, 2005), the EBMR, Cochrane Register, and bibliographies of the published articles. Three investigators extracted data regarding intervention, outcomes, and effect size. A total of 456 patients was included in this analysis, with periodontal treatment as predictor and the actual change in hemoglobin A1c level as the outcome. The weighted average decrease in actual HbA1c level was $0.38 \%$ for all studies, $0.66 \%$ when restricted to type 2 diabetic patients, and $0.71 \%$ if antibiotics were given to them. However, none was statistically significant.
\end{abstract}

\section{Keywords}

meta-analysis; inflammatory mediators; hemoglobin A1c; non-surgical periodontal treatment; antibiotics treatment

\section{INTRODUCTION}

Over 20 million Americans have diabetes mellitus, with one million new patients being diagnosed each year (ADA, 2005). Inflammatory processes involving pro-inflammatory cytokines, C-reactive protein, and TNF- $\alpha$ have been implicated in the pathogenesis of diabetes mellitus. Several dental researchers have postulated that IL-1 $\beta$, IL-6, and CRP from periodontal infection might contribute to the total inflammatory burden (Craig et al., 2003; D'Aiuto et $a l ., 2005)$. One early study showed that exodontia (removing teeth with advanced decay), a source of possible inflammatory mediators, improved glycemic control (Williams and Mahan, 1960).

\footnotetext{
*corresponding author, sjanket@post.harvard.edu.

A supplemental appendix to this article is published electronically only at http://www.dentalresearch.org.
} 
Since Williams' and Mahan's study, over 60 studies have examined the relationship between oral infection and glycemic control among diabetic patients. However, most of them were cross-sectional studies where causal association could not be inferred, and not all published reports were in agreement. These results have been summarized in several reviews (Grossi and Genco, 1998; Taylor, 2001, 2003), and only one review assessed longitudinal studies where causal association might be assumed, but the results were summarized without quantification (Taylor, 2003). Thus, we commenced this meta-analysis to review all published evidence systematically and to quantify the impact of periodontal treatment on HbA1c. Further, we explored the possible causes for the discrepant reports. We tested the null hypothesis that "periodontal treatment does not affect glycemic control in patients with diabetes".

\section{MATERIALS \& METHODS}

\section{Searching}

Two investigators searched MEDLINE for all published articles in English with periodontal disease, diabetes mellitus, glycemic control, and HbA1c as search terms, and 'exploded' these terms further. Additional searches were conducted, with the same search terms, in Evidencebased Medicine Reviews by the American College of Physicians Journal Club and Cochrane Central Register of Controlled Trials. We combined these terms and limited our search to humans and the English language. We restricted the search to post-1980, to avoid changes in secular trends. From the abstracts, we selected longitudinal studies as preliminary candidates, including both observational and intervention studies; full texts of preliminary candidate studies were obtained. Three researchers reviewed the full texts independently, then decided collectively on each paper's eligibility for inclusion. We then restricted our sample to intervention studies to quantify the effects of periodontal treatment.

\section{Selection}

The inclusion criteria were as follows:

1. Original investigation of intervention where causal inferences may be made.

2. Duration of the study of at least 2 months.

3. Primary or secondary outcome was a measure of glycemic control, i.e., HbA1c level, and the predictor was periodontal treatment.

4. Published between 1980 and January, 2005, in the English language.

\section{Data Abstraction}

From each included study, three investigators independently extracted data regarding the study design, diabetes type, number of participants, duration of follow-up, treatment or intervention given, outcomes measures, confounding adjustment, and the results.

\section{Study Characteristics}

Most studies provided basic non-surgical periodontal therapy as the intervention. Some studies supplemented non-surgical periodontal debridement with chlorhexidine oral rinse or low-dose tetracycline or amoxicillin, systemically (Grossi et al., 1997; Rodrigues et al., 2003) or locally (Iwamoto et al., 2001). Most studies reported change in HbA1c in \%, but some studies reported the number of subjects at different levels of HbA1c (Christgau et al., 1998). In such cases, we calculated the mean HbA1c by multiplying the number of subjects by the median value of that category, then dividing by the total number of subjects. Two studies reported an extremely small standard deviation (Grossi et al., 1997; Stewart et al., 2001). For these studies, we 
imputed the mean standard deviation of all studies included in this meta-analysis, assuming homogeneity according to the Q-statistic.

\section{Quantitative Data Synthesis}

Utilizing Stata version 8.0, we estimated the treatment effect for individual trials by calculating the differences in mean $\mathrm{HbA} 1 \mathrm{c}$ levels between the pre- and post-periodontal treatments. We assessed heterogeneity by the Q-statistic, and publication bias using a funnel plot and Egger's and Begg's tests (Egger et al., 2001). We calculated the standard error of the weighted mean difference by pooling the variances in both groups, assuming equal variances on the basis of Q-statistic and taking the square root. We pooled data using the inverse variance method of weighting (for continuous outcomes) and using a random-effects model (DerSimonian and Laird, 1986). Additionally, to assess the effect differences due to diabetes type, the type of control (i.e., self as control or parallel control), or the treatment modality (i.e., non-surgical debridement $v s$. antibiotics administration), we conducted meta-regressions and stratified analyses.

\section{RESULTS}

\section{Trial Flow}

The flow diagram for the search process responsive to the consensus statement to Improve the Quality of Report of Meta-analysis (QUOROM) (Moher et al., 1999) is presented in Fig. 1. The detailed search process is illustrated in the APPENDIX (Appendix Table 1).

\section{Study Characteristics}

We identified 370 titles from our search, and from these titles we selected 64 abstracts. All three investigators read 64 abstracts individually and selected 23 preliminary candidate studies. Full texts of these 23 studies were obtained and evaluated. Collectively, we selected 11 intervention studies as final candidates satisfying our inclusion criteria set a priori (Table 1) (Miller et al., 1992;Seppala and Ainamo, 1994;Aldridge et al., 1995;Smith et al., 1996; Westfelt et al., 1996;Grossi et al., 1997;Christgau et al., 1998;Iwamoto et al., 2001;Stewart et al., 2001; Rodrigues et al., 2003). One of these 11 studies (Smith et al., 1996) could not provide a numeric value of $\mathrm{HbA} 1 \mathrm{c}$ and was excluded from analyses. Studies considered but excluded in this review and the reasons for exclusion are listed in the APPENDIX (Appendix Table 2).

\section{Publication Bias}

Egger's and Begg's tests yielded p-values of 0.69 and 0.81, respectively, indicating no consistent evidence of quantitative publication bias. In addition, the funnel plot demonstrated no clear visual evidence of bias (Appendix Fig.).

\section{Heterogeneity}

The Q-statistic for testing heterogeneity was not significant. For all 10 studies, the Q-statistic $=0.32$; for type 2 diabetes studies only $(\mathrm{N}=5), \mathrm{Q}=0.18(\mathrm{p}=0.99)$. Thus, all included studies were quite homogeneous, and fixed-effects and random-effects models yielded identical results.

\section{Validity Assessment}

Two reviewers evaluated each study's quality independently. The agreement rate was $89 \%$. Agreement was defined as the difference in total quality scores between the reviewers being within one standard deviation. Criteria used for quality assessment were based on our previous quality score rubrics, modified to accommodate clinical trials in periodontal treatment (Janket et al., 2003) (Appendix Table 3). 


\section{Quantitative Data Synthesis}

Ten studies involving 456 type 1 and type 2 diabetic patients resulted in statistically nonsignificant decrease of $\mathrm{HbA} 1 \mathrm{c}$ levels by $0.38 \%$ (Fig. 2). The light and black squares designate the results from type 1 and type 2 diabetic patients, respectively, and a dotted square designates results from a type 1 and type 2 mixed group. Visual review of the graph suggests that there was a correlation between the diabetes type and the direction of outcomes. That is, the research that investigated type 2 diabetes mellitus (DM) appeared to generate stronger effects of periodontal treatment on $\mathrm{HbA} 1 \mathrm{c}$ reduction than did the studies that examined predominantly the type $1 \mathrm{DM}$ group. The result of meta-regression showed a non-significant decrease of $0.57 \%$ among type $2 \mathrm{DM}$ patients only. The type of control did not manifest any substantial difference in the level of HbA1c $(\mathrm{p}=0.82)$. The stratified analysis suggested that antibiotic treatment conferred $0.27 \%$ lower HbA1c level than that conferred by non-surgical debridement $(-0.71 \%$ vs. $-0.44 \%$ ) (Table 2, Fig. 2). Overall, the reduction in HbA1c levels was not significant, and we cannot reject the null hypothesis that "periodontal treatment does not affect glycemic control in diabetic patients".

\section{DISCUSSION}

Over all, this meta-analysis of 10 intervention studies found a non-significant $0.38 \%$ reduction in actual value of $\mathrm{HbAlc}$, and $0.71 \%$ among 5 studies conducted among patients with type 2 diabetes. However, inflammation is implicated in the pathogenesis of type 2 diabetes; thus, including subjects with type 1 diabetes, where the pathogenesis is presumed to be an autoimmune process (Ludwig and Ebbeling, 2001), might have attenuated the effects of periodontal treatment on glycemic control. Thus, restricting the summary results among type 2 diabetic patients may be more pertinent. When each study has a very small sample size, a meta-analysis may offer a better estimate of periodontal treatment than any individual study.

Some studies might have considered their mean as a population mean and used standard errors of the means rather than standard deviation (Grossi et al., 1997; Stewart et al., 2001). Thus, their standard deviations were much smaller than those reported in other studies (standard deviations in parentheses): [Miller '92 (1.85), Seppala '94 (1.77), Aldridge '95 (2.00), Westfelt '96 (2.00), Grossi '97 (0.6), Christgau '98 (1.6), Stewart '01 (0.6), Iwamoto '01 (1.72), Rodrigues ' 03 (1.75)]. To avoid erroneously amplifying the impact of these studies on the final summary of this meta-analysis, we imputed the average standard deviation of all the studies included in this

Some studies were criticized for dispensing doxycycline systemically, and thus for potential bias due to the reduction of systemic CRP and other systemic pro-inflammatory mediators. Our results suggest, however, that doxycycline $10 \mathrm{mg}$, topically delivered in periodontal pockets (Iwamoto et al., 2001), was more efficacious (10.5\% decrease from pre-treatment level) than systemic administration of doxycycline $100 \mathrm{mg} /$ day (4.7\% decrease from pretreatment levels) in reducing HbA1c (Grossi et al., 1997). In addition, we expected that local administration would have less serious and less frequent adverse events. These facts were not known prior to our systematic review and meta-analysis, and future investigators may wish to consult our recommendations in their respective studies.

Sixty percent of our samples studied predominantly type $1 \mathrm{DM}$ patients who might not have displayed any noticeable changes in HbA1c, due to etiology and tighter control of insulin and $\mathrm{HbA1c}$. Thus, restricting the summary results to type 2 diabetic patients who were not on an insulin regimen (we do not have this information) might elicit the true effects of periodontal treatment. 
Studies with parallel comparison groups tended to show evidence of unbalanced randomization (Grossi et al., 1997; Rodrigues et al., 2003). Clearly, the control group had better glycemic control at baseline, which was significant (Rodrigues et al., 2003). In this case, adjusting for the baseline differences may be appropriate to differentiate the treatment effects from the effects due to baseline characteristics.

Recommendations for future studies are as follows:

1. Type 1 diabetes is presumed to originate from an autoimmune process (Ludwig and Ebbeling, 2001), and is usually controlled by the administration of insulin. Glucose levels in these patients are very tightly monitored and adjusted frequently to prevent hypoglycemic crisis. Thus, any obvious change in HbAlc might not be evident, although it is possible that insulin requirements might have been lower. Further, type 1 diabetic patients in general may be too young to develop moderate to severe periodontitis. Thus, future study participants should be limited to type 2 diabetic patients on oral hypoglycemic agents or diet regimen only.

2. Since both diabetes and periodontal disease are multifactorial, other confounding factors for TNF- $\alpha$ and CRP—-such as smoking, BMI, and diet, as well as baseline characteristics affecting glycemic control—should be adjusted, especially when there are signs of unbalanced randomization. The exception to this principle may apply in cases where 'self' was considered as the control, and the duration of the study was reasonably short (8-12 wks). In this case, BMI change might be negligible, and if diet and smoking status did not change, no adjustment may be justified.

3. Our post hoc calculation of sample size revealed that we needed at least 246 participants to observe a $10 \%$ reduction in $\mathrm{HbA1c}(0.7 \%$ in actual value of $\mathrm{HbA} 1 \mathrm{c})$ with $90 \%$ power. A $10 \%$ reduction in mean $\mathrm{HbA} 1 \mathrm{c}$ is so minute that a large sample size is needed for any significance to be observed. Therefore, $90 \%$ power should be used in sample size calculation.

4. HbA1c reflects the mean glucose level 2-3 mos prior to the measurement of HbA1c. Thus, duration should be at least 2 mos if any relevant changes in HbAlc are to be observed.

5. Comparing the effect of periodontal therapy in diabetic patients and healthy controls incorporates bias due to effect-modification. Diabetes has been associated with heightened immune response (Firatli, 1997; Beck et al., 1998). Thus, the intervention should be rendered only to diabetic patients with the same type, similar duration after diagnosis, and similar baseline glycemic control.

6. The intervention should result in a clear improvement in periodontal health. Ineffective interventions may not be different from non-intervention. Also, amoxicillin administration, when causative agents are predominantly Gram-negative organisms, may elicit less efficacious results (Rodrigues et al., 2003). Local doxycycline treatment targeted to periodontal tissue appeared to be most effective in reducing blood HbA1c levels (Iwamoto et al., 2001). We postulated that local delivery of doxycycline would be associated with fewer adverse events. Our assumption was confirmed when we contacted the investigators of the study where doxycycline was delivered locally. They observed no adverse events among 13 patients in a monthlong trial (personal communication via e-mail with Drs. Nishimura/Iwamoto). However, since this observation was drawn from a single study, further corroboration by future studies is necessary before we can make clear recommendations. Although sample size was larger $(\mathrm{N}=140)$, the VA dental diabetes study documented adverse events with systemic doxycycline administration in $19 \%$ of the participants, and 
$16.4 \%$ ( $86 \%$ of all adverse events) showed mild to severe gastro-intestinal irritation (Jones et al., 2005).

Periodontal therapy with antibiotics appeared to decrease $\mathrm{HbA} 1 \mathrm{c}$ levels by statistically nonsignificant $0.71 \%$ among patients with type 2 diabetes. Although this percent improvement in glycemic control may be of value to some patients, the evidence currently available was not strong enough for us to reject the null hypothesis, "periodontal treatment does not affect glycemic control in patients with diabetes". Further studies with a larger sample size among appropriate target populations and utilizing effective treatments are needed to discern whether there is a significant clinical benefit of periodontal therapy on blood sugar control in persons with type 2 diabetes.

\section{Acknowledgements}

Dr. Jones is supported by VA HSR \& D grant \#DII99.206-1 and by Boston University. Dr. Van Dyke is supported by NIH grant DE13191.

\section{References}

ADA. 2005. Diabetes Statistics. American Diabetes Association Web site, http://www.diabetes.org/diabetes-statistics.jsp

Aldridge JP, Lester V, Watts TL, Collins A, Viberti G, Wilson RF. Single-blind studies of the effects of improved periodontal health on metabolic control in type 1 diabetes mellitus. J Clin Periodontol 1995;22:271-275. [PubMed: 7622632]

Beck JD, Offenbacher S, Williams R, Gibbs P, Garcia R. Periodontitis: a risk factor for coronary heart disease? Ann Periodontol 1998;3:127-141. [PubMed: 9722697]

Christgau M, Palitzsch KD, Schmalz G, Kreiner U, Frenzel S. Healing response to non-surgical periodontal therapy in patients with diabetes mellitus: clinical, microbiological, and immunologic results. J Clin Periodontol 1998;25:112-124. [PubMed: 9495610]

Craig RG, Yip JK, So MK, Boylan RJ, Socransky SS, Haffajee AD. Relationship of destructive periodontal disease to the acute-phase response. J Periodontol 2003;74:1007-1016. [PubMed: 12931763]

D'Aiuto F, Nibali L, Parkar M, Suvan J, Tonetti MS. Short-term effects of intensive periodontal therapy on serum inflammatory markers and cholesterol. J Dent Res 2005;84:269-273. [PubMed: 15723869]

DerSimonian R, Laird N. Meta-analysis in clinical trials. Control Clin Trials 1986;7:177-188. [PubMed: 3802833]

Egger, M.; Smith, GD.; Altman, DG. Systematic reviews in health care: meta-analysis in context. 2nd ed. London: BMJ; 2001.

Firatli E. The relationship between clinical periodontal status and insulin-dependent diabetes mellitus. Results after 5 years. J Periodontol 1997;68:136-140. [PubMed: 9058330]

Grossi SG, Genco RJ. Periodontal disease and diabetes mellitus: a two-way relationship. Ann Periodontol 1998;3:51-61. [PubMed: 9722690]

Grossi SG, Skrepcinski FB, DeCaro T, Robertson DC, Ho AW, Dunford RG, et al. Treatment of periodontal disease in diabetics reduces glycated hemoglobin. J Periodontol 1997;68:713-719. [PubMed: 9287060]

Iwamoto Y, Nishimura F, Nakagawa M, Sugimoto H, Shikata K, Makino H, et al. The effect of antimicrobial periodontal treatment on circulating tumor necrosis factor-alpha and glycated hemoglobin level in patients with type 2 diabetes. J Periodontol 2001;72:774-778. [PubMed: 11453240]

Janket SJ, Baird AE, Chuang SK, Jones JA. Meta-analysis of periodontal disease and risk of coronary heart disease and stroke. Oral Surg Oral Med Oral Pathol Oral Radiol Endod 2003;95:559-569. [PubMed: 12738947]

Jones JA, Garcia RI, Wehler C, Rich S, Miller D, Rothendler J, et al. Recruitment and baseline characteristics: the VA dental diabetes study (VA-DDS) (abstract). J Dent Res 2005;84(Spec Iss A): 2584.(www.dentalresearch.org) 
Ludwig DS, Ebbeling CB. Type 2 diabetes mellitus in children: primary care and public health considerations. J Am Med Assoc 2001;286:1427-1430.

Miller LS, Manwell MA, Newbold D, Reding ME, Rasheed A, Blodgett J, et al. The relationship between reduction in periodontal inflammation and diabetes control: a report of 9 cases. J Periodontol 1992;63:843-848. [PubMed: 1403592]

Moher D, Cook DJ, Eastwood S, Olkin I, Rennie D, Stroup DF. Improving the quality of reports of metaanalyses of randomised controlled trials: the QUOROM statement. Quality of reporting of metaanalyses. [See comment]. Lancet 1999;354:1896-1900. [PubMed: 10584742]

Rodrigues DC, Taba MJ, Novaes AB, Souza SL, Grisi MF. Effect of non-surgical periodontal therapy on glycemic control in patients with type 2 diabetes mellitus. J Periodontol 2003;74:1361-1367. [PubMed: 14584871]

Seppala B, Ainamo J. A site-by-site follow-up study on the effect of controlled versus poorly controlled insulin-dependent diabetes mellitus. J Clin Periodontol 1994;21:161-165. [PubMed: 8157767]

Smith GT, Greenbaum CJ, Johnson BD, Persson GR. Short-term responses to periodontal therapy in insulin-dependent diabetic patients. J Periodontol 1996;67:794-802. [PubMed: 8866319][erratum in J Periodontol 67:1368, 1996].

Stewart JE, Wager KA, Friedlander AH, Zadeh HH. The effect of periodontal treatment on glycemic control in patients with type 2 diabetes mellitus. J Clin Periodontol 2001;28:306-310. [PubMed: 11314885]

Taylor GW. Bidirectional interrelationships between diabetes and periodontal diseases: an epidemiologic perspective. Ann Periodontol 2001;6:99-112. [PubMed: 11887478]

Taylor GW. The effects of periodontal treatment on diabetes. J Am Dent Assoc 2003;134(10 Suppl):41s48s. [PubMed: 18196672]

Westfelt E, Rylander H, Blohme G, Jonasson P, Lindhe J. The effect of periodontal therapy in diabetics. Results after 5 years. J Clin Periodontol 1996;23:92-100. [PubMed: 8849844]

Williams RC Jr, Mahan CJ. Periodontal disease and diabetes in young adults. J Am Med Assoc 1960;172:776-778. 


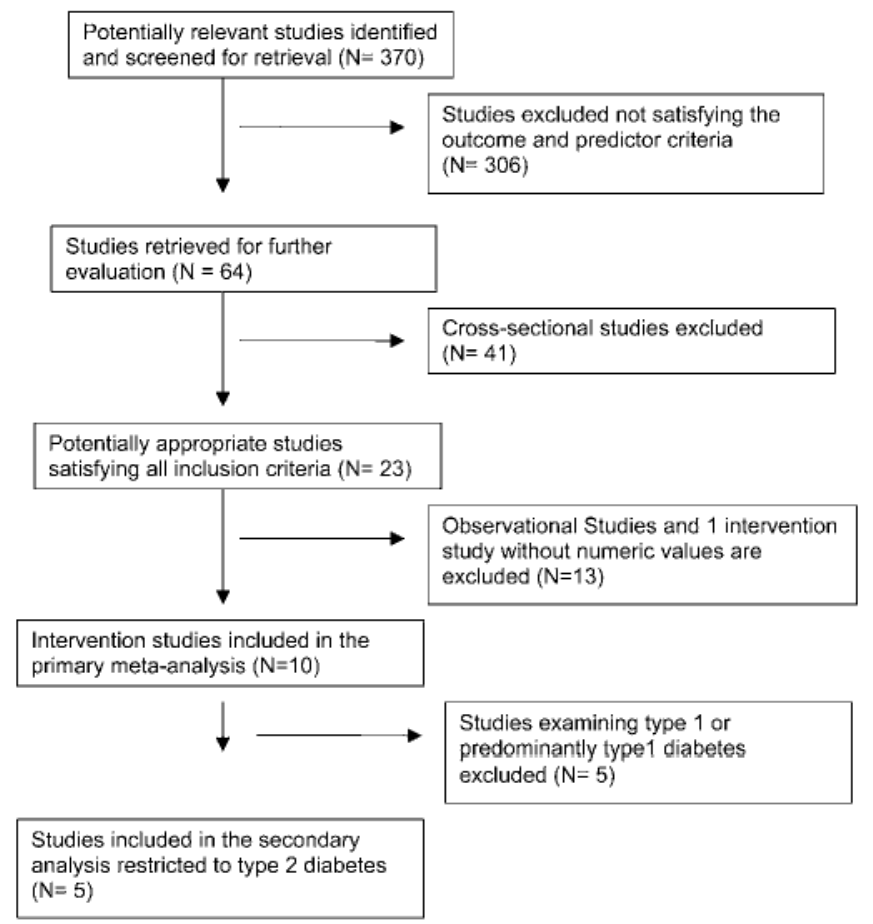

Figure 1.

Flow chart. 


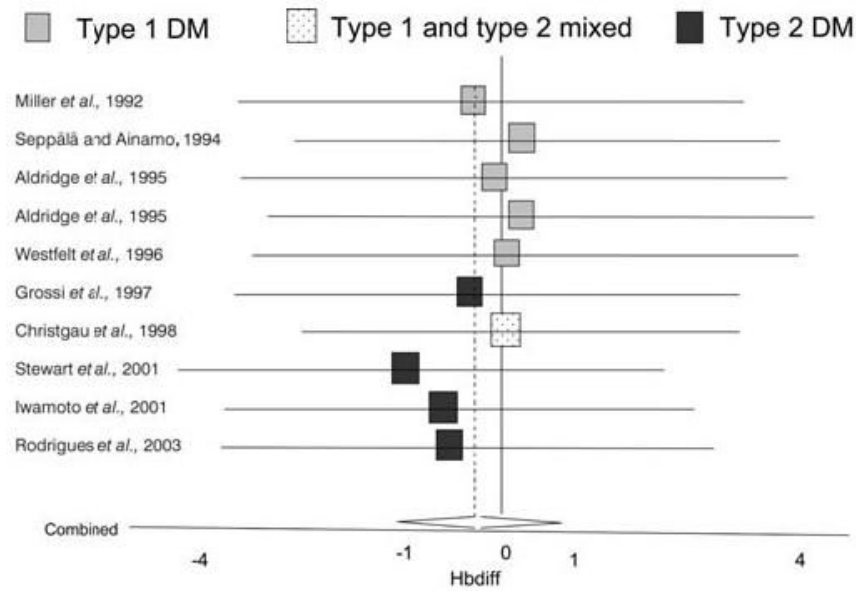

Figure 2.

Effects of periodontal treatment on HbA1c. Hbdiff = change in HbA1c meta-analysis. 


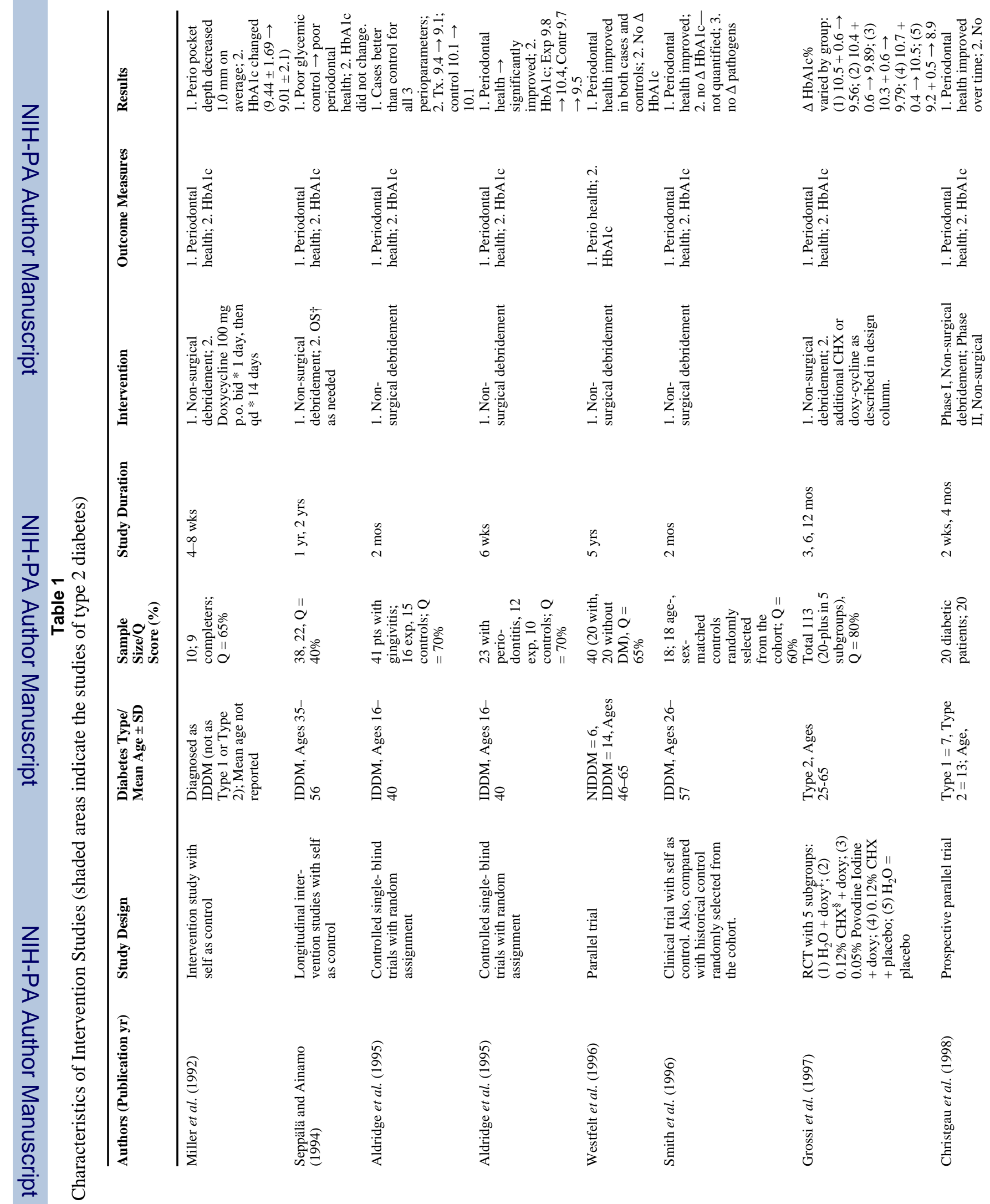


Janket et al.

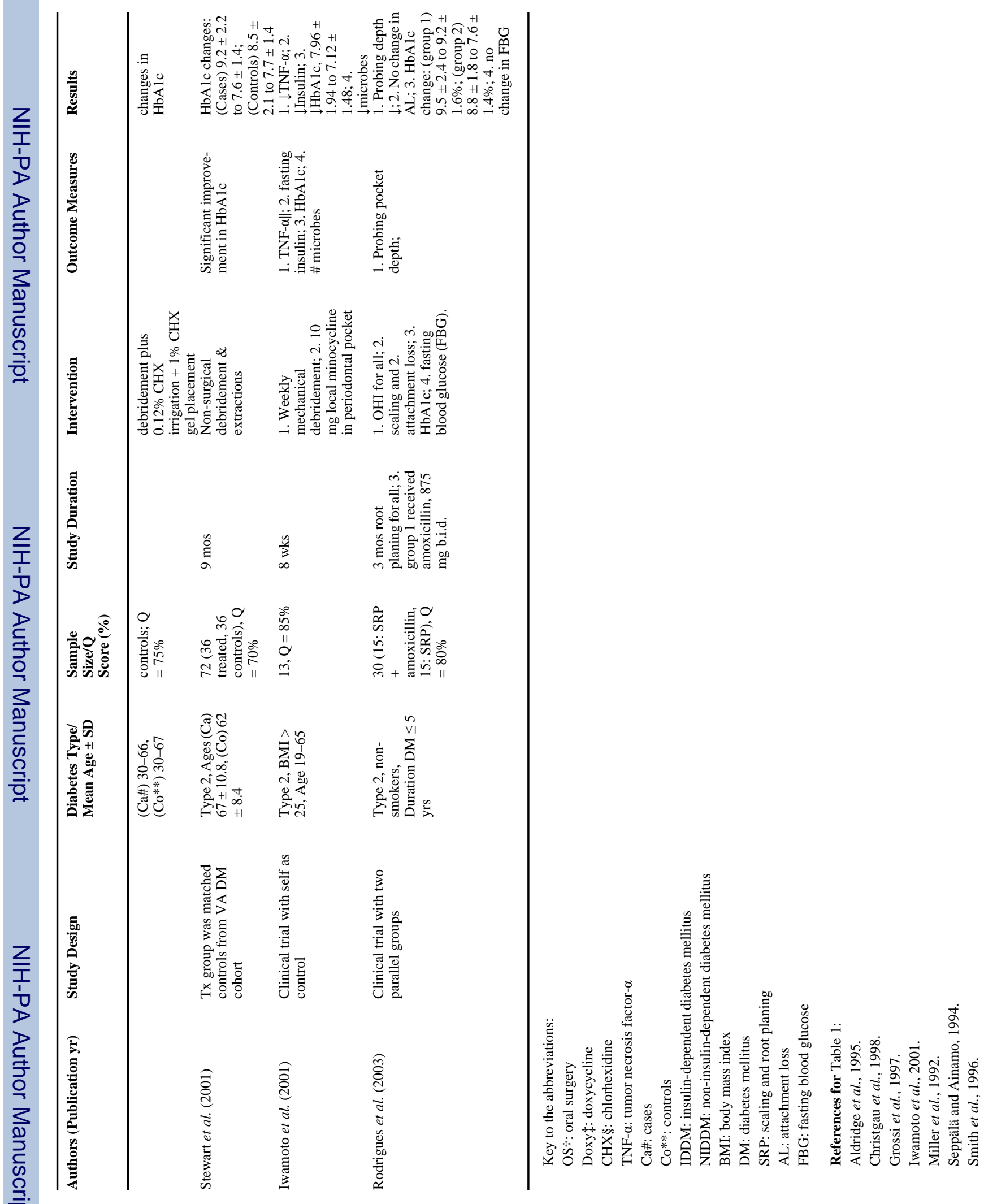


ป 
Table 2

Quantitative Summary of Periodontal Treatment on HbA1c

\begin{tabular}{lcc}
\hline & $\begin{array}{c}\text { Weighted Average Change in } \\
\text { HbA1c }\end{array}$ & 95\% Confidence Interval \\
\hline All intervention studies & $-0.4 \%$ & $-1.5,0.7$ \\
Studies of type 2 DM only & $-0.7 \%$ & $-2.2,0.9$ \\
Non-surgical debridement only & $-0.4 \%$ & $-2.1,1.3$ \\
Antimicrobial intervention in type 2 diabetic patients & $-0.7 \%$ & $-2.3,0.9$ \\
\hline
\end{tabular}

